\title{
DETERMINANTS OF READMISSION AFTER COMPULSORY INPATIENT TREATMENT
}

S. Pinto ${ }^{1}$, A. Machado ${ }^{1}$, M.E. Caseiro-Alves ${ }^{1}$, F. Andrade ${ }^{1}$, I. Soares da Costa ${ }^{1}$, J. Morais ${ }^{1}$, R. Guedes ${ }^{1}$, A. Silva ${ }^{1}$, C. Silveira ${ }^{1}$, R. Curral ${ }^{1}$, R. Coelho ${ }^{1}$.

${ }^{1}$ Centro Hospitalar e Universitário São João, Clínica de Psiquiatria e Saúde Mental, Porto, Portugal.

\section{INTRODUCTION}

The compulsory admission of individuals with psychiatric disorders is a protective measure whenever mental severe patients refuse treatment, allowing an effective clinical follow-up in a community setting. However, clinical and psychosocial determinants may hinder the success of the treatment plan, leading to hospital readmissions. To better address this issue, we aim to identify pre- and postdischarge characteristics associated with short term readmission in compulsorily treated inpatients.

\section{RESULTS}

Readmission was significantly associated $(p<0,05)$ :

- non-attendance to psychiatry appointment after discharge

previous compulsory ambulatory treatment

discharge diagnosis after first admission (F20-F29)

\section{OBJECTIVES}

To study determinants for the readmission of previously compulsory treated inpatients.

\section{MATERIALS AND METHODS}

Retrospective observational study of clinical and social and demographic data from every inpatient compulsory treatment episode between January 1st2016 and December 31st2018 in the Psychiatry Service of São João University Hospital Center, with hospital readmissions until 90 days. Statistical analysis was done using SPSS.

\begin{tabular}{|c|c|c|c|c|c|c|}
\hline $\begin{array}{c}\text { Number of } \\
\text { readmissions }\end{array}$ & Gender & Age & Marrital Status & Education & Household & Working Status \\
\hline 55 & $\begin{array}{c}69,1 \% \text { Male } \\
30,9 \% \text { Female }\end{array}$ & $\begin{array}{c}18-80 \text { yo. } \\
\text { (Mean } 40,33 \text { yo) }\end{array}$ & $\begin{array}{c}\text { 70,9\% Single } \\
\text { 12,7\% Married } \\
\text { 14,5\% Divorced } \\
\text { 1,8\% Widowed }\end{array}$ & $\begin{array}{c}\text { None }-15,5 \% \\
4 \text { years }-21,8 \% \\
5-9 \text { years }-30,9 \% \\
10-12 \text { years }-12,7 \% \\
>12 \text { years }-20 \%\end{array}$ & $\begin{array}{c}\text { Homeless }-7,3 \% \\
1-25,5 \% \\
2 \text { or more }-67,3 \%\end{array}$ & $\begin{array}{c}\text { Employed - 12,7\% } \\
\text { Unemployed - 54,5\% } \\
\text { Retired - 25,5\% } \\
\text { Student - 5,5\% }\end{array}$ \\
\hline
\end{tabular}

\begin{tabular}{|c|c|c|c|c|}
\hline $\begin{array}{c}\text { Number of } \\
\text { readmissions }\end{array}$ & $\begin{array}{c}\text { LAl-AP } \\
\text { treatment after } \\
\text { first discharge }\end{array}$ & $\begin{array}{c}\text { Number of days until post- } \\
\text { discharge appointment } \\
\text { (after 1st admission) }\end{array}$ & $\begin{array}{c}\text { Attendance to } \\
\text { post-discharge } \\
\text { appointment }\end{array}$ & $\begin{array}{c}\text { Substance } \\
\text { Abuse }\end{array}$ \\
\hline 55 & $49 \%$ & $\begin{array}{c}\text { Minimum - 3 days } \\
\text { Maximum - 64 days } \\
\text { Mean - 24 days }\end{array}$ & $\begin{array}{c}36,7 \% \text { Yes } \\
63,3 \% \text { No }\end{array}$ & $\begin{array}{c}36,7 \% \text { Yes } \\
63,3 \% \text { No }\end{array}$ \\
\hline
\end{tabular}

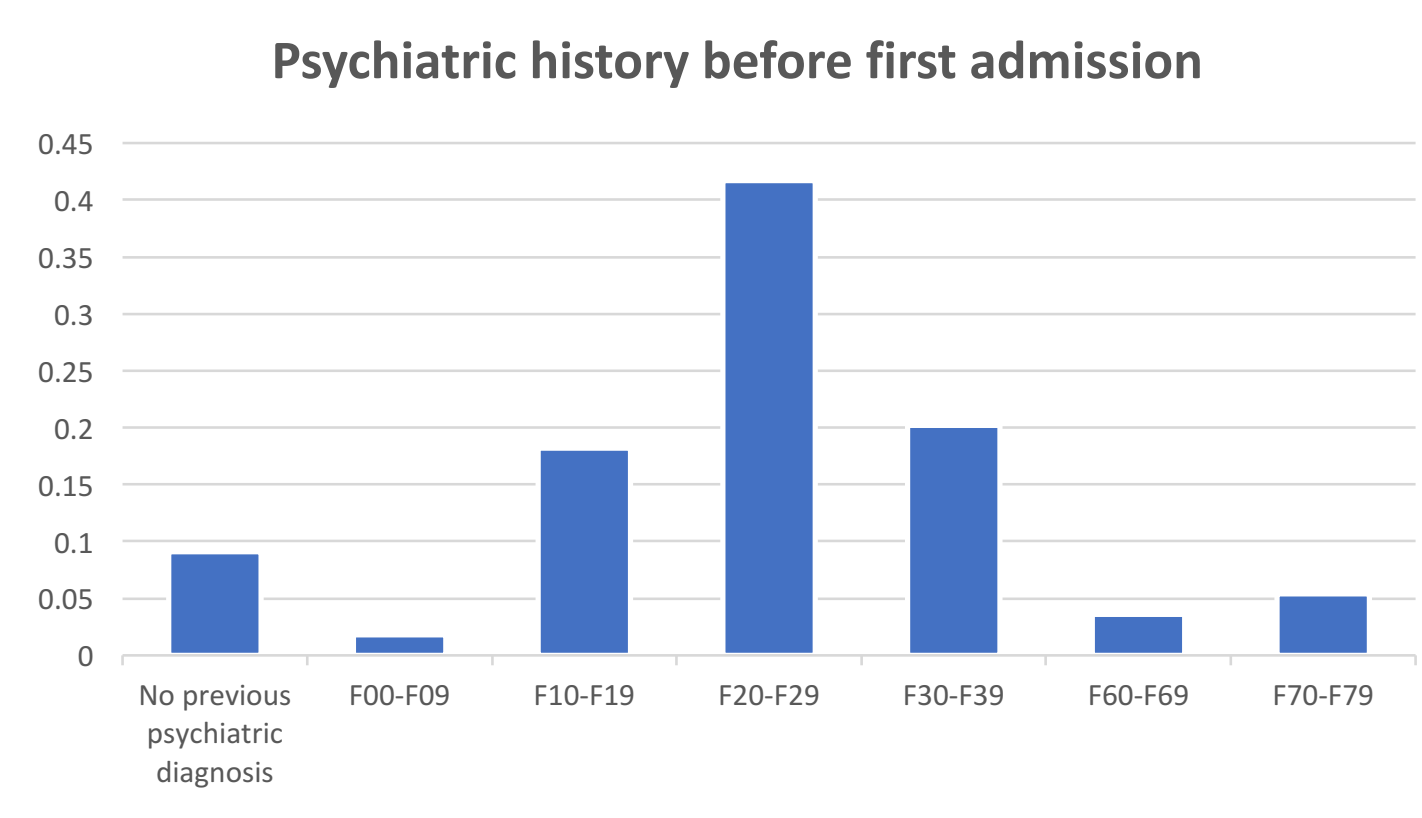

Diagnosis at discharge from readmission

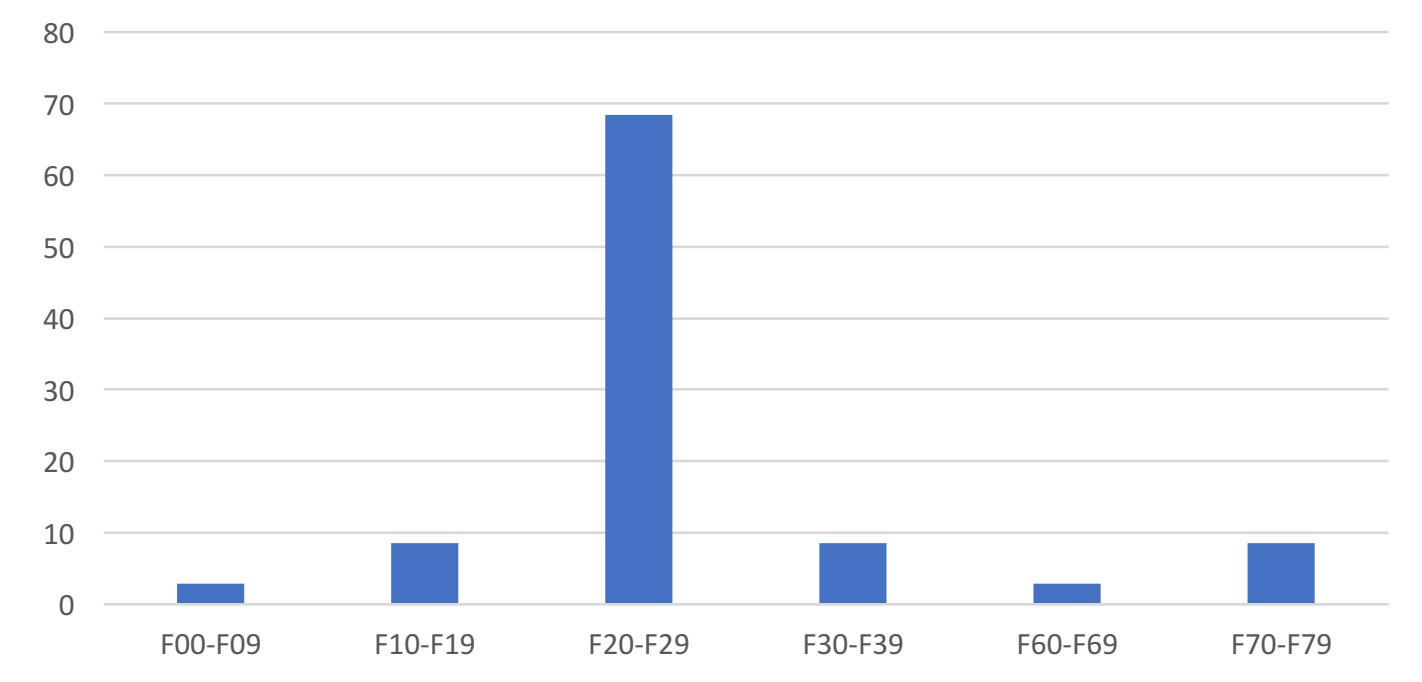

Readmissions per period post-discharge

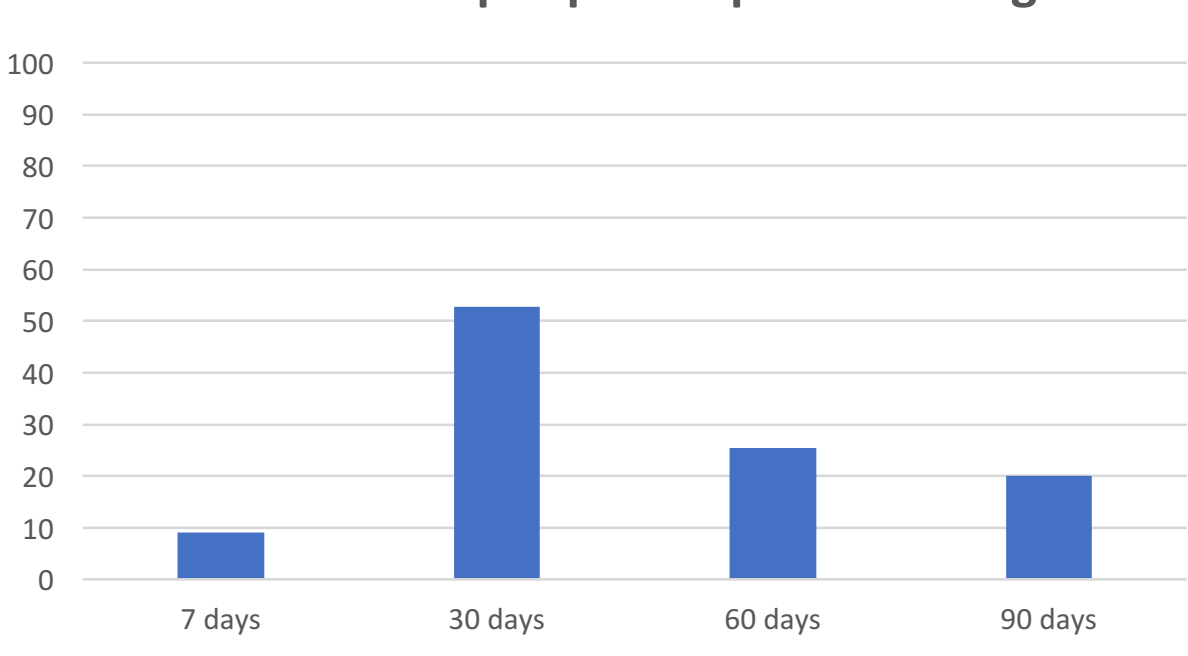

Type of readmission

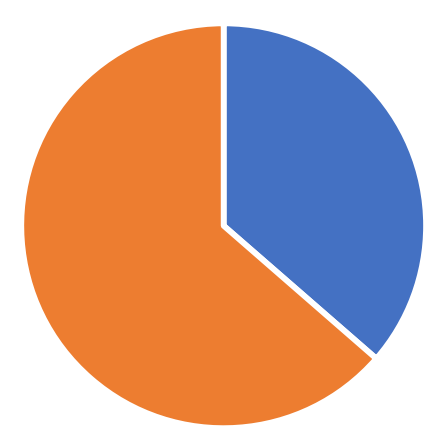

- Voluntary - Compulsory
Type of discharge postreadmission

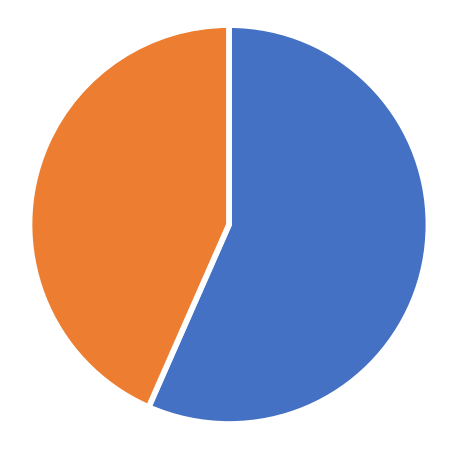

\section{CONCLUSIONS}

A closer post-discharge follow-up seems to play a more relevant role than non-modifiable factors in these patients' rate of readmission. Regular home visits could play an important role in achieving this goal. Further studies should investigate the impact of substance abuse on the readmission rate. 